\title{
GENERALIZED INVERSES AND SPECTRAL THEORY
}

\author{
BY
}

\author{
CONSTANTIN APOSTOL AND KEVIN CLANCEY
}

\begin{abstract}
The concept of a generalized spectral projection associated with a subset in the semi-Fredholm domain of a bounded operator on a Hilbert space is introduced. These generalized spectral projections possess many of the desirable properties of spectral projections associated with spectral sets. In particular, generalized spectral projections are used to separate finite sets of singular points from the semi-Fredholm domain.
\end{abstract}

This note is concerned with some spectral theory questions in the semi-Fredholm domain of a bounded operator $T$ acting on a Hilbert space. In the semiFredholm domain of $T$ there exist at most a countable collection of complex numbers $\lambda$ where the projection onto the kernel of $\lambda-T$ is discontinuous. These points, referred to as the singular points in the semi-Fredholm domain, do not accumulate inside the semi-Fredholm domain. The points $\lambda$ where the projection onto the kernel of $\lambda-T$ is continuous are called regular points.

In the study of several questions connected with approximation in the algebra of all bounded operators, it is often necessary to separate from the semi-Fredholm domain a finite number of these singular eigenvalues. This means one decomposes $T$ into a direct sum of two operators $T_{1} \oplus T_{2}$, where $T_{1}$ contains the singular behavior of $T$ and this finite set of singular eigenvalues are regular points for $T_{2}$. A procedure for accomplishing this separation is described in [3]. In this note an alternate procedure for the separation of a finite set of singular eigenvalues is given.

The method entails constructing spectral projections for singular eigenvalues in the semi-Fredholm domain. It should be observed that these singular eigenvalues need not be isolated points in the spectrum and, therefore, one cannot construct the spectral projections in the usual manner. Fortunately, there is a convenient $3 \times 3$ triangular representation of an operator which efficiently displays the spectral properties of the operator in the semi-Fredholm domain [3]. This leads to questions about generalized inverses and generalized spectral projections for $3 \times 3$ triangular matrices of operators. The basic properties of these generalized inverses and generalized spectral projections are developed in this note. These properties should be of independent interest. 
Allan [1], [2] has shown how to construct an analytic right inverse for an operator $T$ on a domain where $\lambda-T$ is surjective. This result of Allan allows a more canonical construction of the generalized spectral projections associated with singular eigenvalues. The authors would like to thank Professor I. C. Gohberg for drawing their attention to the work of Allan.

The note is organized as follows. $\S 1$ contains preliminaries and notations. In $\S 2$ triangular generalized inverses are constructed for $3 \times 3$ triangular matrices of operators. Also in this section the basic properties of these generalized inverses and generalized spectral projections are described. In $\S 3$ the triangular matrix model for an operator in the semi-Fredholm domain is described. The results of $\S 2$ are then applied to construct a lattice homomorphism from the family of finite subsets of singular points in the semi-Fredholm domain to the lattice of all projection operators. This construction allows one to easily separate a finite set of these singular points.

1. Preliminaries. Let $H$ be a complex Hilbert space and $L(H)$ the algebra of all bounded operators on $H$. For $T \in L(H)$ the notations $\sigma(T)$ and $\sigma_{e}(T)$ will be used for the spectrum and essential spectrum of $T$, respectively. The corresponding resolvent sets will be denoted by $\rho(T)$ and $\rho_{e}(T)$. It is convenient to also introduce the notations $\rho_{r}(T)$ and $\rho_{l}(T)\left(\rho_{r e}(T), \rho_{l e}(T)\right)$ for the right and left (essential) resolvents of $T$, respectively.

An operator $T \in L(H)$ is called a Fredholm operator in case $0 \in \rho_{e}(T)$. In case $0 \in \rho_{r e}(T) \cup \rho_{l e}(T)$, the operator $T$ is called a semi-Fredholm operator. The semi-Fredholm domain of $T$ is the set $\rho_{s-F}(T)=\rho_{r e}(T) \cup \rho_{l e}(T)$.

The kernel or null space of an operator $T$ will be written $\operatorname{ker} T$ and $P_{\text {ker }} T$ will be employed for the orthogonal projection of $H$ onto the kernel of $T$. The initial space of the operator $T$ is the range of the operator $I-P_{\text {ker }} T$ and the final space is the closure of the range of $T$.

Let $T$ be an operator with closed range. An operator $F \in L(H)$ will be called a generalized inverse for $T$ in case, $T F$ is a projection onto the final space of $T$ and $F T$ is a projection onto the initial space. Unless the operator $T$ is invertible, a generalized inverse of an operator with closed range is not unique.

Let $T \in L(H)$ and $G$ an open subset in the complex plane such that the operator $\lambda-T$ has closed range for every $\lambda \in G$. An operator valued function $F$ : $G \rightarrow L(H)$ will be called a generalized inverse function for $T$ in $G$, in case for $\lambda \in G, F(\lambda)$ is a generalized inverse for $\lambda-T$. If, in addition, the operator valued function $F$ is continuous and, for each pair $\lambda, \mu$ in a component of $G$, the resolvent identity

$$
(F(\lambda)-F(\mu)) /(\lambda-\mu)=-F(\lambda) F(\mu)
$$

holds, then $F$ will be called a generalized resolvent for $T$ in $G$. 
It is clear that if $F$ is a generalized resolvent for an operator $T$ in an open set $G$, then $F$ is analytic on $G$. On the other hand, not every generalized inverse function, which is analytic on a component of an open set verifies the resolvent equation (1) on this component.

In case $G \subset \rho_{r}(T)$, then a generalized inverse (resolvent) is called a right-inverse (resolvent) for $T$ in $G$. Similar definitions are made for left inverses and left resolvents. Allan [1], [2] has shown that there exists an analytic right inverse function in all of $\rho_{r}(T)$ (of course, a similar result is true for left inverses in $\rho_{l}(T)$ ). It is apparently not known if there exists a right resolvent in all of $\rho_{r}(T)$. It is easy to see, however, that locally right resolvents exist in $\rho_{r}(T)$.

Let $T \in L(H)$. A point $\lambda$ at which the mapping $\mu \rightarrow P_{\operatorname{ker}(\mu-T)}$ is continuous is called a regular point for the operator $T$. Otherwise, $\lambda$ is called a singular point. The set of singular points in the semi-Fredholm domain is denoted by $\rho_{s-F}^{s}(T)$. It is known [3] that $\rho_{s-F}^{s}(T)$ coincides with the discontinuities of the function

$$
m(\lambda)=\text { minimum dimension }\left\{\operatorname{ker}(\lambda-T), \operatorname{ker}(\lambda-T)^{*}\right\} .
$$

This is an at most countable set which does not accumulate in $\rho_{s-F}(T)$ (see, e.g. [3], Gohberg and Krein [4], and Kato [5]). It is easy to see that $\rho_{s-F}^{s}(T) \cap$ $\rho_{r}(T)=\varnothing$.

Suppose $\lambda$ is an isolated point in the spectrum of the operator $T$. Let $P(\{\lambda\})$ be the spectral projection of $T$ corresponding to the spectral set $\{\lambda\}$. The dimension of the spectral space $P(\{\lambda\}) H$ will be denoted by $\operatorname{sp} \operatorname{dim}(\lambda ; T)$. The collection of all isolated eigenvalues $\lambda$ of $T$ for which $\operatorname{sp} \operatorname{dim}(\lambda ; T)$ is finite will be denoted by $\sigma_{p}^{0}(T)$. Clearly, $\sigma_{p}^{0}(T) \subset \rho_{s-F}^{s}(T)$.

2. Generalized inverses for triangular matrices of operators. In this section we assume that the Hilbert space $H$ has the direct sum decomposition $H=H_{r}+$ $H_{0}+H_{l}$, where $H_{r}, H_{0}, H_{l}$ are closed subspaces. It is further assumed that relative to this decomposition of $H$, the operator $T$ has the triangular form

$$
T=\left(\begin{array}{lll}
T_{r} & A & B \\
0 & T_{0} & C \\
0 & 0 & T_{l}
\end{array}\right)
$$

Let $G$ be an open set. Assume that $T_{r}$ has a right inverse function $R$, and $T_{l}$ has a left inverse function $L$ in $G$. Further assume that $\lambda-T_{0}$ is invertible for $\lambda$ in $G$ with resolvent $R\left(\lambda: T_{0}\right)$. For $\lambda$ in $G$, set

$$
F(\lambda)=\left(\begin{array}{ccc}
R(\lambda) & R(\lambda) A R\left(\lambda: T_{0}\right) & R(\lambda)\left[A R\left(\lambda: T_{0}\right) C+B\right] L(\lambda) \\
0 & R\left(\lambda: T_{0}\right) & R\left(\lambda: T_{0}\right) C L(\lambda) \\
0 & 0 & L(\lambda)
\end{array}\right)
$$


The operator valued function $F$ can easily be seen to have the following properties:

(i) $F$ is a generalized inverse function for $T$ in $G$.

(ii) If $R, L$ are analytic in $G$, then $F$ is an analytic generalized inverse function for $T$ in $G$.

(iii) If $R, L$ are right and left resolvents, of $T_{r}, T_{l}$ respectively, then $F$ is a generalized resolvent of $T$ in $G$.

Next suppose that $G$ is an open subset of the complex plane and that $R, L$ are analytic right and left inverse functions in $G$ of $T_{r}, T_{l}$, respectively. Let $\sigma$ be a compact subset of $G$, such that $\sigma \subset \sigma\left(T_{0}\right)$ and $G_{0}=G \backslash \sigma$ is a subset of $\rho\left(T_{0}\right)$. Let $\Gamma$ be a closed rectifiable Jordan curve in $G_{0}$ which surrounds $\sigma$ and has positive orientation with respect to that component of the plane determined by $\Gamma$ containing the set $\sigma$. Let $F$ be the analytic generalized inverse function for $T$ in $G_{0}$ defined by (3). Form the generalized spectral projection of $T$ associated with $\sigma$ by $P(\sigma)=(2 \pi i)^{-1} \int_{\Gamma} F(\lambda) d \lambda$. The operator $P(\sigma)$ has the form:

(4) $P(\sigma)=\left(\begin{array}{ccc}0 & \frac{1}{2 \pi i} \int_{\Gamma} R(\lambda) A R\left(\lambda: T_{0}\right) d \lambda & \frac{1}{2 \pi i} \int_{\Gamma} R(\lambda) A R\left(\lambda: T_{0}\right) C L(\lambda) d \lambda \\ 0 & P_{0}(\sigma) & \frac{1}{2 \pi i} \int_{\Gamma} R\left(\lambda: T_{0}\right) C L(\lambda) d \lambda \\ 0 & 0 & 0\end{array}\right)$; here, $P_{0}(\sigma)=(2 \pi i)^{-1} \int_{\Gamma} R\left(\lambda: T_{0}\right) d \lambda$ is the usual spectral projection of $H_{0}$ onto the spectral subspace for $T_{0}$ corresponding to the spectral set $\sigma$.

The generalized spectral projection $P(\sigma)$ has many of the usual properties of a spectral projection:

(a) $P^{2}(\sigma)=P(\sigma)$.

(b) $P(\sigma) T=T P(\sigma)$.

(c) Let $T_{\sigma}$ be the restriction of $T$ to the invariant subspace $P(\sigma) H$. Then $\sigma\left(T_{\sigma}\right)=\sigma$.

(d) Let $P^{\prime}(\sigma)=I-P(\sigma)$ and $T_{\sigma}^{\prime}$ be the restriction of $T$ to the invariant subspace $P^{\prime}(\sigma) H$. Then every point in $G$ is a regular point for $T_{\sigma}^{\prime}$.

(e) Suppose that $\sigma=\sigma_{1} \cup \sigma_{2}$, where $\sigma_{1}$ and $\sigma_{2}$ are disjoint compact sets. Form $P\left(\sigma_{1}\right), P\left(\sigma_{2}\right)$ by integrating $F(\lambda)$ around closed curves $\Gamma_{1} \subset G_{0}, \Gamma_{2} \subset G_{0}$ which surround only the part of $\sigma$ labelled $\sigma_{1}, \sigma_{2}$, respectively. Then $P\left(\sigma_{1}\right) P\left(\sigma_{2}\right)=P\left(\sigma_{2}\right) P\left(\sigma_{1}\right)=0$ and $P(\sigma)=P\left(\sigma_{1}\right)+P\left(\sigma_{2}\right)$.

(f) Assume that $\sigma=\left\{\lambda_{i}\right\}_{i=1}^{n} \subset \sigma_{p}^{0}\left(T_{0}\right)$ where the $\lambda_{i}$ are distinct. Then

and

$$
\operatorname{dimension}(P(\sigma) H)=\sum_{i=1}^{n} \operatorname{sp} \operatorname{dim}\left(\lambda_{i} ; T_{0}\right)
$$

$$
\operatorname{sp} \operatorname{dim}\left(\lambda_{i} ; T_{\sigma}\right)=\operatorname{sp} \operatorname{dim}\left(\lambda_{i} ; T_{0}\right), \quad i=1, \ldots, n
$$


In the remainder of this section we give brief indications of the proofs of properties (a)-(f).

Proof of (a). This is a matrix computation. In the 1,3 position in the matrix of $P^{2}(\sigma)$ there appears the operator

$$
E=(2 \pi i)^{-2} \int_{\Gamma} R(\lambda) A R\left(\lambda: T_{0}\right) d \lambda \int_{\Gamma} R\left(\mu: T_{0}\right) C L(\mu) d \mu .
$$

Enlarge $\Gamma$ to a contour $\Gamma^{\prime} \subset G_{0}$ which contains $\Gamma$ in its interior. Then

$$
\begin{aligned}
E= & (2 \pi i)^{-2} \int_{\Gamma} \int_{\Gamma^{\prime}} R(\lambda) A R\left(\lambda: T_{0}\right) R\left(\mu: T_{0}\right) C L(\mu) d \mu d \lambda \\
= & (2 \pi i)^{-2} \int_{\Gamma} \int_{\Gamma^{\prime}} R(\lambda) A R\left(\lambda: T_{0}\right)(\mu-\lambda)^{-1} C L(\mu) d \mu d \lambda \\
& -(2 \pi i)^{-2} \int_{\Gamma} \int_{\Gamma^{\prime}} R(\lambda) A R\left(\mu: T_{0}\right)(\mu-\lambda)^{-1} C J(\mu) d \mu d \lambda \\
= & (2 \pi i)^{-1} \int_{\Gamma} R(\lambda) A R\left(\lambda: T_{0}\right) C L(\lambda) d \lambda \\
& -(2 \pi i)^{-2} \int_{\Gamma^{\prime}}\left[\int_{\Gamma} R(\lambda)(\mu-\lambda)^{-1} d \lambda\right] A R\left(\lambda: T_{0}\right) C L(\mu) d \mu \\
= & (2 \pi i)^{-1} \int_{\Gamma} R(\lambda) A R\left(\lambda: T_{0}\right) C L(\lambda) d \lambda .
\end{aligned}
$$

This last operator is the 1,3 entry in $P(\sigma)$. It is important to note that only the analyticity of $R, L$ was used in the above computation. The verification of $P^{2}(\sigma)=P(\sigma)$ for the other entries in the matrices proceeds similarly.

Proof OF (b). This is another matrix computation. Again we will compare only the 1,3 entries in $P(\sigma) T$ and $T P(\sigma)$. The check of the equality of the other entries in the matrices $P(\sigma) T$ and $T P(\sigma)$ is left to the reader.

The 1,3 entry in $P(\sigma) T$ is the operator

$$
\begin{aligned}
E & =(2 \pi i)^{-1} \int_{\Gamma} R(\lambda) A R\left(\lambda: T_{0}\right) C d \lambda+(2 \pi i)^{-1} \int_{\Gamma} R(\lambda) A R\left(\lambda: T_{0}\right) C L(\lambda) T_{l} d \lambda \\
& =(2 \pi i)^{-1} \int_{\Gamma} \lambda R(\lambda) A R\left(\lambda: T_{0}\right) C L(\lambda) d \lambda .
\end{aligned}
$$

The 1, 3 entry in $\operatorname{TP}(\sigma)$ is the operator

$$
\begin{aligned}
E^{\prime} & =(2 \pi i)^{-1} \int_{\Gamma} T_{r} R(\lambda) A R\left(\lambda: T_{0}\right) C L(\lambda)+(2 \pi i)^{-1} \int_{\Gamma} A R\left(\lambda: T_{0}\right) C L(\lambda) d \lambda \\
& =(2 \pi i)^{-1} \int_{\Gamma} \lambda R(\lambda) A R\left(\lambda: T_{0}\right) C L(\lambda) d \lambda .
\end{aligned}
$$

Obviously, the 1,3 entries in $P(\sigma) T$ and $T P(\sigma)$ are equal.

Proof of (c). Let $\mu \notin \sigma$. Choose a closed contour $\Gamma^{\prime}$ in $G_{0}$ containing $\sigma$ such that $\mu$ is not inside $\Gamma^{\prime}$. Set $S(\mu)=(2 \pi i)^{-1} \int_{\Gamma^{\prime}} F(\lambda)(\mu-\lambda)^{-1} d \lambda$. Then $S(\mu)(\mu-T) P(\sigma)=P(\sigma)$ and $P(\sigma)(\mu-T) S(\mu)=P(\sigma)$. It follows that $\mu \notin \sigma\left(T_{\sigma}\right)$. 
The inclusion $\sigma \subset \sigma\left(T_{\sigma}\right)$ is not difficult to obtain.

Proof of (d). A computation shows that $\operatorname{ker}\left(\lambda-T_{\sigma}^{\prime}\right)=\operatorname{ker}\left(\lambda-T_{r}\right)$, for $\lambda \in G$. This establishes (d).

Proof of (e). The identity $P(\sigma)=P\left(\sigma_{1}\right)+P\left(\sigma_{2}\right)$ follows from the analyticity of $F$ in $G_{0}$. To complete the proof of (e) it suffices to establish that $P\left(\sigma_{1}\right) P\left(\sigma_{2}\right)=0$. Consider the 1,3 entry in $P\left(\sigma_{1}\right) P\left(\sigma_{2}\right)$. It is the operator

$$
\begin{aligned}
E & =(2 \pi i)^{-2} \int_{\Gamma_{1}} R(\lambda) A R\left(\lambda: T_{0}\right) d \lambda \int_{\Gamma_{2}} R\left(\mu: T_{0}\right) C L(\mu) d \mu \\
& =(2 \pi i)^{-2} \int_{\Gamma_{1}} R(\lambda) A R\left(\lambda: T_{0}\right) P_{0}\left(\sigma_{1}\right) d \lambda \int_{\Gamma_{2}} P_{0}\left(\sigma_{2}\right) R\left(\mu: T_{0}\right) C L(\mu) d \mu .
\end{aligned}
$$

It follows from the fact that $P_{0}\left(\sigma_{1}\right) P_{0}\left(\sigma_{2}\right)=0$, that $E=0$. The verification of $P\left(\sigma_{1}\right) P\left(\sigma_{2}\right)=0$ for the other entries in $P\left(\sigma_{1}\right) P\left(\sigma_{2}\right)$ proceeds similarly.

REMARK. The assumption that $F$ was analytic in $G_{0}$ was not actually necessary in the last argument. If one knows that an analytic generalized inverse exists separately in open sets $G_{1}, G_{2}$ which surround $\sigma_{1}, \sigma_{2}$, respectively, then still one has $P\left(\sigma_{1}\right) P\left(\sigma_{2}\right)=P\left(\sigma_{2}\right) P\left(\sigma_{1}\right)=0$.

ProOF OF (f). In virtue of property (e) it suffices to establish this result only in the case where $\sigma=\{\lambda\} \subset \sigma_{p}^{0}\left(T_{0}\right)$. In fact it suffices to establish that

$$
\operatorname{dimension}(P(\{\lambda\}) H)=\operatorname{dimension}\left(P_{0}(\{\lambda\}) H_{0}\right) .
$$

It is clear that dimension $\left(P_{0}(\{\lambda\}) H_{0}\right) \leqslant \operatorname{dimension}(P(\{\lambda\}) H)$. Further, $P(\{\lambda\}) H \subset$ $H_{r}+H_{0}, P(\{\lambda\}) H_{r}=(0)$, and, therefore,

$$
\operatorname{dimension}(P(\{\lambda\}) H) \leqslant \operatorname{dimension}\left(P_{0}(\{\lambda\}) H_{0}\right) \text {. }
$$

Also, $P(\{\lambda\}) H_{0}=P(\{\lambda\}) P_{0}(\{\lambda\}) H_{0}$, and we obtain dimension $(P(\{\lambda\}) H) \leqslant$ dimension $\left(P_{0}(\{\lambda\}) H_{0}\right)$. This completes the proof.

3. The lattice of generalized spectral projections. This section begins with a description of a triangular form for an operator $T \in L(H)$ when $H$ is decomposed in a natural way relative to the semi-Fredholm domain. This triangular form plus its basic spectral properties appear in [3].

For $T \in L(H)$ the collection of regular points in the semi-Fredholm domain will be denoted by $\rho_{s-F}^{r}(T)$. Following [3] we define

$$
\begin{aligned}
& H_{r}(T)=\text { c.l.m. }\{\operatorname{ker}(\lambda-T)\}_{\lambda \in \rho_{S-F}^{r}(T)}, \\
& H_{l}(T)=\text { c.l.m. }\left\{\operatorname{ker}(\lambda-T)^{*}\right\}_{\lambda \in \rho_{S}^{r}-F(T)}, \\
& H_{0}(T)=H \ominus\left(H_{r}(T)+H_{l}(T)\right) ;
\end{aligned}
$$

here, c.l.m. is an abbreviation for "closed linear manifold". The subspaces $H_{r}(T)$ and $H_{0}(T)$ are orthogonal so that $H_{r}(T)+H_{l}(T)$ is closed.

The restriction of $T$ to the invariant subspace $H_{r}(T)$ is denoted by $T_{r}$, and the compressions of $T$ to the subspaces $H_{l}(T)$ and $H_{0}(T)$ will be denoted by $T_{0}$ 
and $T_{l}$, respectively. Relative to the decomposition $H=H_{r}(T) \oplus H_{0}(T) \oplus$ $H_{l}(T)$, the operator $T$ has the form

$$
T=\left(\begin{array}{lll}
T_{r} & A & B \\
0 & T_{0} & C \\
0 & 0 & T_{l}
\end{array}\right) .
$$

The following result appears in [3]:

THEOREM 1. For $T \in L(H)$ we have

(i) $\rho_{s-F}(T) \subset \rho_{r}\left(T_{r}\right) \cap \rho_{l}\left(T_{l}\right)$,

(ii) $\rho_{s-F}^{r}(T) \subset \rho\left(T_{0}\right)$,

(iii) $\rho_{s-F}^{s}(T) \subset \sigma_{p}^{0}\left(T_{0}\right)$,

(iv) $\sigma_{p}^{0}(T) \subset \rho\left(T_{r}\right) \cap \rho\left(T_{l}\right) \cap \sigma_{p}^{0}\left(T_{0}\right)$, and for $\lambda \in \sigma_{p}^{0}(T)$, sp $\operatorname{dim}(\lambda ; T)=$ $\operatorname{sp} \operatorname{dim}\left(\lambda ; T_{0}\right)$.

Using the results of Allan [1], [2] and property (i) in Theorem 1, it is possible to find an analytic right inverse function $R$ for $T_{r}$ on $\rho_{s-F}(T)$ and an analytic left inverse function $L$ for $T_{l}$ on $\rho_{s-F}(T)$. Set $G_{0}=\rho_{s-F}(T) \backslash \rho_{s-F}^{s}(T)$ and define the analytic generalized inverse function $F$ for $T$ in $G_{0}$ by (3).

Let $T$ denote the lattice of finite subsets of $\rho_{s-F}^{s}(T)$ ordered by inclusion. For each $\sigma \in T$ one can form the generalized spectral projection $P(\sigma)$ from the analytic generalized inverse function $F$ as described in $\S 2$. It is clear from property (e) that the map $\sigma \rightarrow P(\sigma)$ is a lattice homorphism from $T$ into the lattice of projections on $H$.

The following theorem is a summary of many of the basic properties of the generalized spectral projections $P(\sigma)$ for $\sigma \in T$. It also yields Theorem 3.3 of [3] as a corollary.

Theorem 2. Let $T \in L(H)$ and let (5) denote the triangular matrix representation of $T$ relative to the decomposition $H=H_{r}(T) \oplus H_{0}(T) \oplus H_{l}(T)$. Choose an analytic right inverse function $R$ for $T_{r}$ and an analytic left inverse function $L$ for $T_{l}$ in $\rho_{s-F}(T)$. Form the analytic generalized inverse function $F$ for $T$ in $G_{0}=\rho_{s-F}(T) \backslash \rho_{s-F}^{s}(T)$ determined by (3), and let $\sigma \rightarrow P(\sigma)$ be the mapping which associates with $\sigma \in T$ the generalized spectral projection $P(\sigma)$. Then:

(i) The map $\sigma \rightarrow P(\sigma)$ is a lattice homomorphism of $T$ into the lattice of projections on $H$.

(ii) If $H_{\sigma}=P(\sigma) H$, then the collection $\left\{H_{\sigma}: \sigma \in T\right\}$ is a lattice of invariant subspaces for the operator $T$; moreover, $\operatorname{dim} H_{\sigma}=\Sigma_{\lambda \in \sigma} \operatorname{sp} \operatorname{dim}\left(\lambda ; T_{0}\right)$, for $\sigma \in T$.

(iii) If $T_{\sigma}$ denotes the restriction of $T$ to $H_{\sigma}$, then $\sigma\left(T_{\sigma}\right)=\sigma$.

(iv) If $T_{\sigma}^{\prime}$ denotes the restriction of $T$ to $H_{\sigma}^{\prime}=(I-P(\sigma)) H$, then $G_{0} \cup$ $\sigma \subset \rho_{s-F}^{r}\left(T_{\sigma}^{\prime}\right)$. 


\section{REFERENCES}

1. G. R. Allan, On one-sided inverses in Banach algebras of holomorphic vector-valued functions, J. London Math. Soc. 42 (1967), 463-470. MR 35 \#5939.

2. - Holomorphic vector-valued functions on a domain of holomorphy, J. London Math. Soc. 42 (1967), 509-513. MR 35 \#5940.

3. C. Apostol, The correction by compact perturbation of the singular behavior of op. erators (to appear).

4. I. C. Gohberg and M. G. Krein, The basic propositions on defect numbers, root numbers and indices of linear operators, Uspehi Mat. Nauk 12 (1957), no. 2 (74), 43-118; English transl., Amer. Math. Soc. Transl. (2) 13 (1960), 185-264. MR 20 \#3459; 22 \#3984.

5. T. Kato, Perturbation theory for linear operators, Die Grundlehren der math. Wissenschaften, Band 132, Springer-Verlag, New York, 1966. MR 34 \#3324.

INSTITUTE OF MATHEMATICS, BUCHAREST, ROMANIA

DEPARTMENT OF MATHEMATICS, UNIVERSITY OF GEORGIA, ATHENS, GEORGIA 30602 